\title{
Asymmetry and 4D-STEM: When the Phase Object Approximation Is Qualitatively Incorrect
}

\author{
Mark Oxley, Debangshu Mukherjee and Jordan Hachtel \\ Oak Ridge National Laboratory, Oak Ridge, Tennessee, United States
}

The combination of aberration corrected scanning transmission electron microscopy (STEM) and highspeed cameras has led to renewed interest in the field of differential phase contrast (DPC). It has been demonstrated that the variation in the center of mass (COM) is directly proportional to the electric field within the specimen [1]. The divergence of the COM hence provides a direct measure of the charge density from which the incident probe has scattered. The position of the atomic nuclei can be located to high precision using simultaneously acquired annular dark field (ADF) measurements. Given a priori knowledge of the associated Debye-Waller factors the effective nuclear charge density can be calculated, and the electron density can be found. An alternative approach is to reconstruct the scattering potential whose gradient is proportional to the COM displacement [2]. These approaches essentially provide equivalent information, since the relationship between the electrostatic scattering potential and the charge density is well understood.

In Figure 1 we show the electron scattering potential for strontium titanate (STO) calculated using the parameterization of X-ray scattering factors presented by Waasmaier and Kirfel [3] and the Mott-Bethe formula. The total charge density derived from the potential is shown in (b) The calculated nuclear charge density derived from the atomic locations and Debye-Waller factors is shown in (c). The electron charge density obtained by subtracting (c) from (b) is shown in (d). These results do not include the effect of the probe intensity, which is convolved with the experimental results [1] or can be deconvolved to construct the scattering potential [2]. The relationship between the scattering potential and the charge densities is exact, but to derive these quantities from the COM displacements the phase object approximation is usually invoked.

The phase object approximation is only viable for suitably thin specimens, at best a few tens of Ångstroms thick. The number of specimens that can be prepared and examined in this regime is however limited. Many examples in the literature have shown acceptable qualitative agreement between theory and experiment for thicker samples, but these have usually been cases with strong symmetries, such as $\mathrm{STO}$ or $\mathrm{Si}$ in the [011] orientation. In figure 2 we examine the asymmetric case of $\mathrm{ZnS}$ in the [011] orientation. For reference, the structure, potential and electric field derived from the potential are shown in (a), (b) and (c) respectively. Calculated COM deflection maps for thicknesses of 3.8, 38 and $76 \AA$ in (d), (e) and (f). Simulations are for $100 \mathrm{kV}$ incident electrons with a $30 \mathrm{mrad}$ probe forming aperture. The beam is focused in the middle of the crystal. In the phase object approximation, the COM deflection should be the electric field convolved with the probe intensity. This is indeed the case for (d) where the intensity is concentrated about the $\mathrm{Zn}$ column. However, for (e) the S column has similar intensity and in (f) the intensity distribution across the columns is reversed.

Asymmetric samples form an important class of materials. This includes polarization of perovskites, interfaces and defects. Clearly in these cases a more robust approach is required for even qualitative analysis of 4D-STEM datasets. In some cases, a more robust quantum mechanical inversion is possible [4], though this potentially leads to high electron dose rates. Alternatively, electron densities calculated using density functional theory could be used as the starting point for 4D-STEM simulations in order to 
validate experimental results, either by examination or possibly machine learning. Such approaches are essential for truly quantitative analysis of 4D-STEM data sets [5].
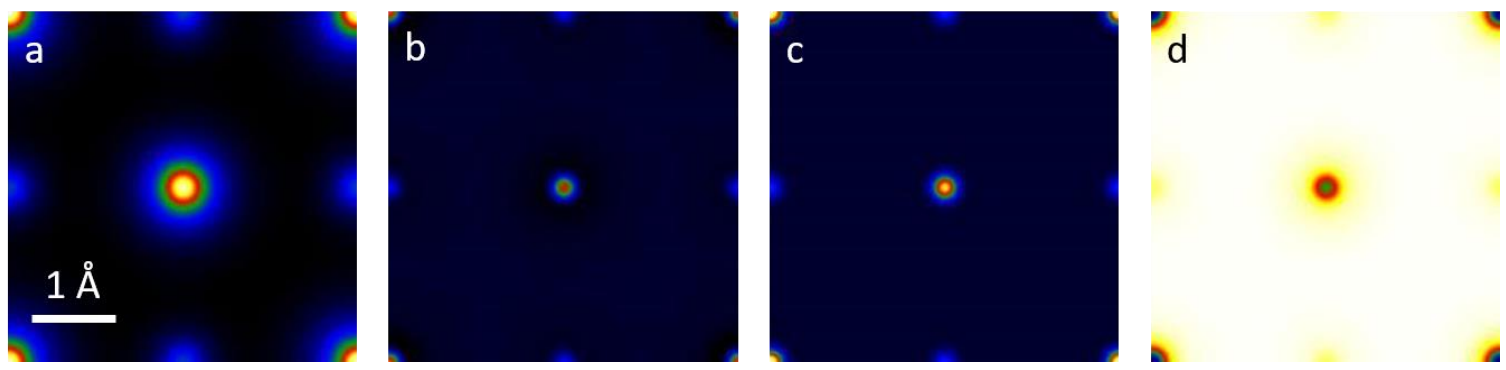

Figure 1. (a) Electron scattering potential of STO for $100 \mathrm{kV}$ incident electrons. Also shown are charge densities (b) Total charge density derived from the potential, (c) Nuclear charge density and (d) Electron charge density from (b) and (c).
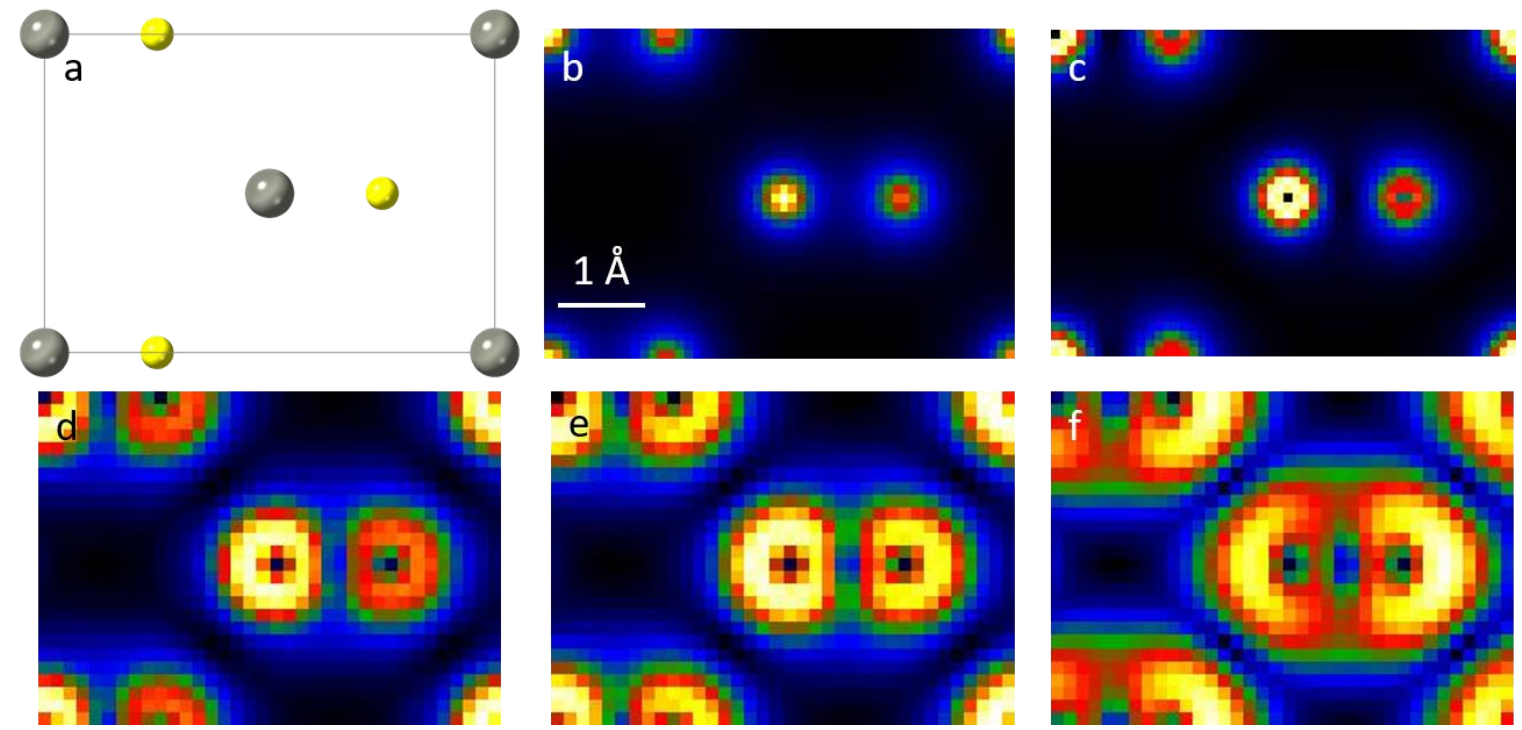

Figure 2. (a) ZnS structure in the [011] orientation. (b) Calculated Potential. (c) Magnitude of electric field from (b). COM deflections for $(\mathrm{d}) \mathrm{t}=3.8 \AA$, (e) $\mathrm{t}=38 \AA$ and (f) $\mathrm{t}=76 \AA$. COM deflections are shown each with their own intensity range to clarify details.

\section{References}

[1] Knut Müller et al., Nature Communications, 5 (2014) 5653.

[2] Ryan Close et al., Ultramicroscopy, 159 (2015) p. 124.

[3] D. Waasmaier and A. Kirfel., Acta Crystallographica Section A, 51 (1995) p. 416.

[4] H. G. Brown et al., Phys. Rev. Lett. 121 (2018) 266102.

[5] This work is supported by the Office of Basic Energy Sciences, Materials Sciences and Engineering Division, U.S. Department of Energy (MPO). Part of this work was performed at the Oak Ridge National Laboratory's Center for Nanophase Materials Sciences (CNMS), a U.S. Department of Energy, Office of Science User Facility (DM,JAH). 